\title{
CMEARTICLE
}

\section{Management of psoriasis in primary care}

Weai Ling $\underline{\operatorname{Lim}}^{1}$, MD, MMed, Choon How $\underline{\mathrm{How}}^{2,3}$, MMed, FCFP, Ki Wei $\underline{\operatorname{Tan}}^{4}$, MRCP, FAMS

\begin{abstract}
John, who always stood out in your waiting room due to his long-sleeved shirt, looked particularly tired today. You saw from the edge of his scalp line that his psoriasis flare was the reason for his attendance. He was embarrassed about his skin condition and struggled with day-to-day life because of it. He tried to avoid dark clothing as it made his shedding, scaly skin more apparent. The shedding skin also made his job as a kitchen helper challenging during food preparation.
\end{abstract}

\section{WHAT IS PSORIASIS?}

Psoriasis is a chronic inflammatory disease with predominant skin, nail and joint involvement. It has a bimodal age of onset (16-22 years and 57-60 years) ${ }^{(1)}$ and affects both sexes equally. ${ }^{(2)}$ Psoriasis is an immune-mediated disease with genetic predisposition. Risk factors and triggers include family history, physical (e.g. skin injury, illness, weather changes) and emotional stress, and alcohol intake.

\section{HOW RELEVANT IS THIS TO MY PRACTICE?}

It is estimated that $2 \%-3 \%$ of the total global population has psoriasis. ${ }^{(3)}$ In Singapore, it is estimated that at least 40,000 persons are affected with psoriasis. ${ }^{(4)}$ Most patients with psoriasis can be managed in the primary care setting with topicals and regular monitoring. A holistic approach is crucial to assess disease severity and the impact on the patients' life, such as on their mood, relationships and social stigma. Screening to look for the presence of joint involvement and comorbidities is useful to provide timely management and better disease outcomes.

\section{WHAT CAN I DO IN MY PRACTICE? Establishing the diagnosis}

The diagnosis is usually clinical and based on the presence of typical erythematous and scaly skin lesions, which may be pruritic. Psoriasis occurs in several distinct clinical forms. Fig. 1 shows the different clinical forms of psoriasis. ${ }^{(5,6)}$ Its distribution usually involves the extensor surfaces (elbows and knees), scalp, nails and sometimes also the intertriginous areas (natal cleft, groin folds and axillae). Box 1 shows the differential diagnoses and their distinguishing clinical features. ${ }^{(7)}$

\section{Psoriatic onychodystrophy}

About $80 \%$ of patients with psoriasis are likely to develop nail psoriasis, or psoriatic onychodystrophy. Nail matrix involvement can result in features such as leuconychia, pitting, red spots in lunulae and crumbling. Nail bed involvement can cause onycholysis, salmon or oil-drop patches, subungual
Box 1. Differential diagnoses and their distinguishing clinical features:

- Atopic dermatitis: predominant symptom of pruritus and typical morphology and distribution (flexural lichenification in adults and older children; facial and extensor papules in infancy)

- Lichen planus: typically pruritic, violaceous papules with frequent mucosal involvement.

- Pityriasis rosea: pink, oval papules and patches with 'Christmas tree' configuration on trunk, presence of a herald patch with sparing of face and distal extremities.

- Tinea corporis: annular scaly patches and plaques with central clearance.

- Onychomycosis: no cutaneous/joint manifestation. Nail scraping for microscopy and culture.

hyperkeratosis and splinter haemorrhages. Nail disease causes aesthetic and functional impairment, and is indicative of more severe forms of psoriasis as well as joint involvement. ${ }^{(8)}$

\section{Psoriatic arthritis}

Up to $40 \%$ of individuals with psoriasis will go on to develop psoriatic arthritis (PsA), usually within 5-10 years of cutaneous disease onset. Regular routine screening of patients with psoriasis is important for early detection of PsA. PsA is characterised by synovitis, enthesitis, dactylitis and spondylitis. ${ }^{(9)}$

\section{Screen for other comorbidities}

Patients with psoriasis are at increased risk of a variety of medical conditions such as depression, anxiety, inflammatory bowel disease, malignancy, metabolic syndrome and cardiovascular disease. ${ }^{(10,11)}$ Hence, it is important to screen patients for other comorbidities.

\section{Management}

An individualised treatment plan is made after incorporating patients' preferences, the disease's impact on quality of life, and the potential benefits and adverse effects of therapies (Fig. 2). ${ }^{(12)}$ Treatment goals include improvement of skin, nails and joint lesions as well as enhancing quality of life.

\footnotetext{
${ }^{1}$ SingHealth Polyclinics - Bedok, ${ }^{2} \mathrm{C}$ are and Health Integration, Changi General Hospital, ${ }^{3}$ Family Medicine Academic Clinical Programme, SingHealth Duke-NUS Academic Medical Centre, ${ }^{4}$ Department of Dermatology, Changi General Hospital, Singapore

Correspondence: Dr Lim Weai Ling, Family Physician, SingHealth Polyclinics - Bedok, Heartbeat@\#02-01, 11 Bedok North Street 1, \#03-01, Singapore 469662 lim.weai.ling@singhealth.com.sg
} 


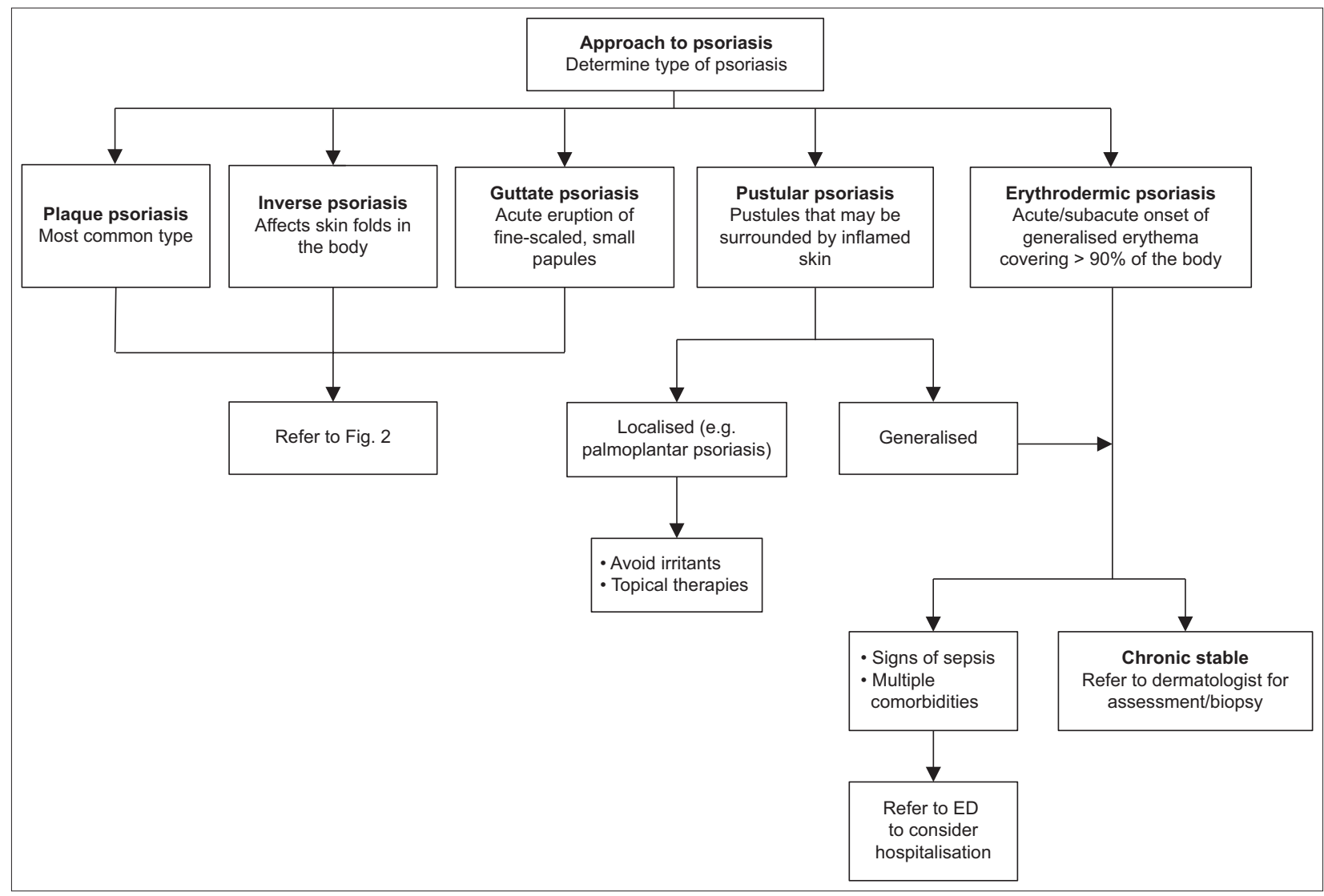

Fig. 1 Flowchart shows the approach to different clinical forms of psoriasis. ED: emergency department

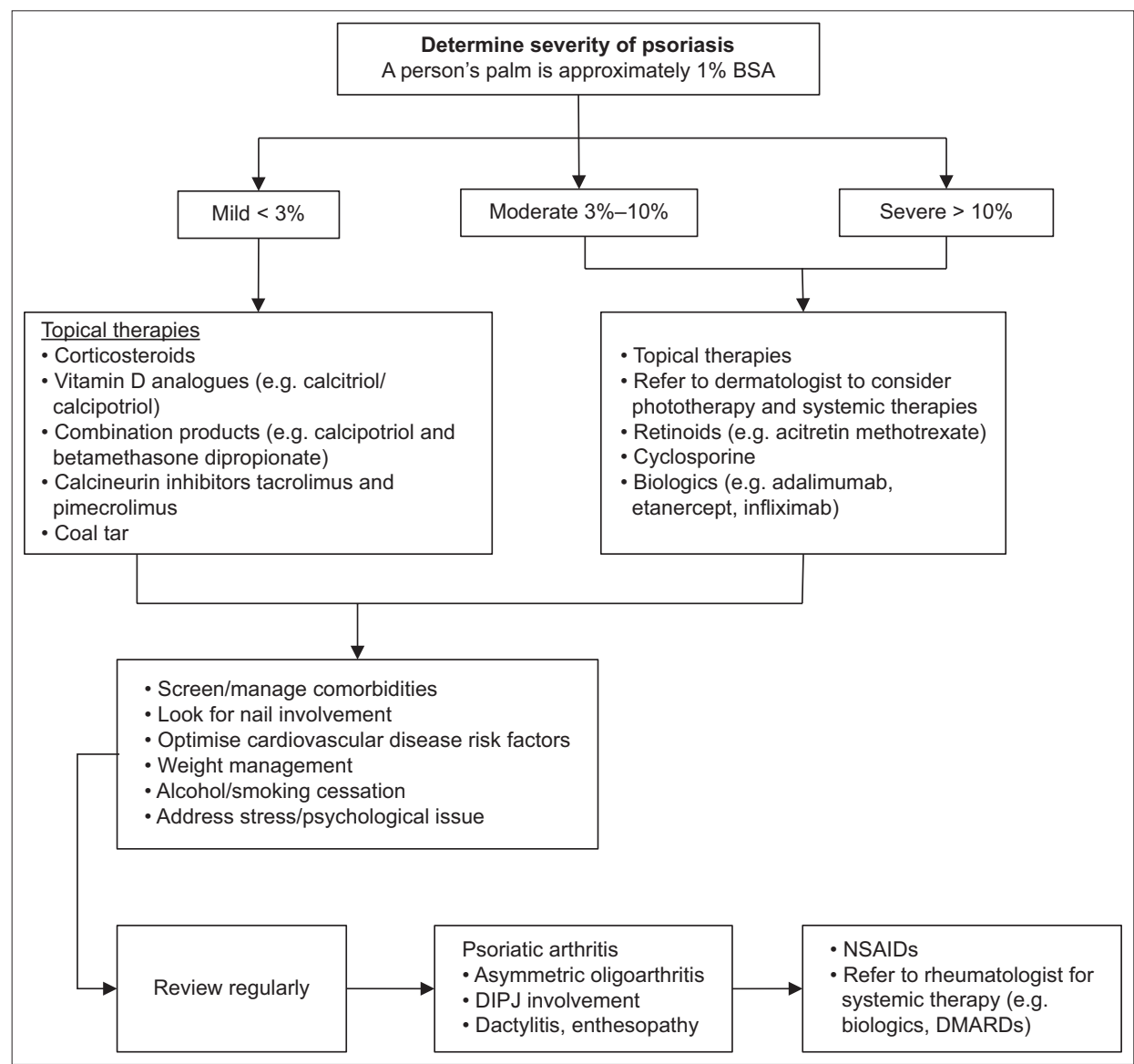

Fig. 2 Flowchart shows the approach to psoriasis of different severities. ${ }^{(12)}$ BSA: body surface area; DIPJ: distal interphalangeal joint; DMARD: diseasemodifying anti-rheumatic drug; NSAID: nonsteroidal anti-inflammatory drug 
Box 2. Indications for specialist referral for patients with psoriasis:

- Poor disease control with appropriate first-line treatments

- Moderate and severe psoriasis requiring phototherapy/systemic

therapies

- Psoriatic arthritis

- Uncertain diagnosis

- Unstable/sepsis in generalised pustular psoriasis or erythrodermic psoriasis

\section{Non-pharmacologic treatment}

Non-pharmacologic management includes identification and avoidance of triggers, stress management, regular use of emollients, smoking and alcohol cessation, and weight management to reduce cardiovascular risk factors.

\section{Pharmacologic treatment}

Topical corticosteroids

Patients with mild to moderate disease can often be treated successfully with topical therapies. Use topical steroids of appropriate potency based on the location and severity of the skin lesions, such as the low-potency topical steroids $1 \%$ hydrocortisone or $0.025 \%$ betamethasone valerate cream on lesions on the face, genitals and flexures. More potent topical steroids can be used for thicker and more persistent lesions on the trunk and limbs.

\section{Topical vitamin D3 analogues}

Calcipotriol, a vitamin D3 analogue, is a first-line topical agent for treatment of plaque psoriasis and moderately severe scalp psoriasis. ${ }^{(6)}$

\section{Combination products}

For local, persistent lesions, potent topical steroids can be used in combination with a vitamin $\mathrm{D}$ analogue.

\section{Systemic therapy}

\section{Phototherapy}

For moderate to severe psoriasis, phototherapy is a mainstay treatment, especially in cases that are unresponsive to topical agents. ${ }^{(6)}$

Systemic therapies such as acitretin, methotrexate, cyclosporine and biologics may be considered.

\section{WHEN SHOULD I REFER TO A SPECIALIST?}

If there are no contraindications, nonsteroidal anti-inflammatory drugs can be used to relieve joint pain in patients with psoriatic arthritis while waiting for a rheumatologist review. Box 2 shows the indications for a referral to a rheumatologist.

\section{USEFUL LINKS}

- $\quad$ About the Psoriasis Association of Singapore: https://www. psoriasis.org.sg/about-us.html (accessed February 18, 2021)

- National Psoriasis Foundation, United States: https://www. psoriasis.org (accessed February 18, 2021)

\section{TAKE HOME MESSAGES}

1. Psoriasis is a chronic inflammatory disease that typically affects the skin and joints. It is also associated with multiple comorbidities including cardiovascular disease, metabolic syndrome and mood problems.

2. Treatment for psoriasis should be individualised and tailored according to the severity of the psoriasis, comorbidities and treatment preferences.

3. Patients should be advised about weight management, reduction of alcohol intake and smoking cessation to reduce cardiovascular risk factors.

4. Patients who do not respond well to topical therapies should be referred to a specialist for early assessment.

5. It is important for physicians to recognise erythrodermic psoriasis and its complications and consider referring the patient to the emergency department if required.

You assessed John's skin condition and noted that he had mild psoriasis with body surface area involvement of about 3\%. Nail pitting was also noted. He did not have joint symptoms. You explained the diagnosis of psoriasis and that there are multiple effective treatments to control the disease. You reassured John that psoriasis is neither contagious nor infectious. He was given aqueous cream moisturiser, betamethasone valerate $1 \%$ scalp lotion and $0.1 \%$ betamethasone valerate cream for the lesions on his limbs. You encouraged him to speak to his co-workers to help them understand his situation. You also referred him to join the Psoriasis Association of Singapore's support group.

\section{REFERENCES}

1. Griffiths CE, Barker JN. Pathogenesis and clinical features of psoriasis. Lancet 2007; 370:263-71.

2. Levine D, Gottlieb A. Evaluation and management of psoriasis: an internist's guide. Med Clin North Am 2009; 93:1291-303.

3. National Psoriasis Foundation. Psoriasis Statistics. Available at: www.psoriasis. org/content/statistics. Accessed May 12, 2020.

4. Psoriasis Association of Singapore. About psoriasis. Available at: www.psoriasis. org.sg/about-psoriasis. Accessed September 20, 2020.

5. National Psoriasis Foundation. Locations and types. Available at: https://www. psoriasis.org/locations-and-types. Accessed September 18, 2020.

6. Canadian Psoriasis Guidelines Committee. Canadian guidelines for the management of plaque psoriasis. Ottawa, ON: Canadian Dermatology Association; 2009.

7. Kim WB, Jerome D, Yeung J. Diagnosis and management of psoriasis. Can Fam Physician 2017; 63:278-85

8. Schons KR, Knob CF, Murussi N, et al. Nail psoriasis: a review of the literature. An Bras Dermatol 2014; 89:312-7.

9. Mease PJ, Armstrong AW. Managing patients with psoriatic disease: the diagnosis and pharmacologic treatment of psoriatic arthritis in patients with psoriasis. Drugs 2014; 74:423-41

10. Kimball AB, Gladman D, Gelfand JM, et al. National Psoriasis Foundation clinical consensus on psoriasis comorbidities and recommendations for screening. J AM Acad Dermatol 2008; 58:1031-42.

11. Chan WMM, Yew YW, Theng TSC, Liew CF, Oon HH. Prevalence of metabolic syndrome in patients with psoriasis: a cross-sectional study in Singapore. Singapore Med J 2020; 61:194-9.

12. Gold LS. How do topical treatments fit into the long-term management of psoriasis across the severity spectrum. Medscape Education Dermatology [online]. Available at: https://www.medscape.org/viewarticle/932576. Accessed February 16, 2021. 


\section{SINGAPORE MEDICAL COUNCIL CATEGORY 3B CME PROGRAMME} (Code SMJ 202103A)

1. Psoriasis is a chronic inflammatory disease with predominant skin, nail and joint involvement.

2. Most patients with psoriasis can be managed in the primary care setting with topicals and regular monitoring.

3. The diagnosis of psoriasis is usually clinical and based on the presence of typical erythematous and scaly skin lesions, which may be pruritic.

4. Psoriasis occurs in several distinct clinical forms, such as plaque psoriasis, guttate psoriasis, inverse psoriasis, pustular psoriasis and erythrodermic psoriasis.

5. Psoriatic onychodystrophy is uncommon and indicative of less severe forms of psoriasis.

6. Nail scraping for microscopy and culture can differentiate psoriatic onychodystrophy from onychomycosis.

7. Psoriatic onychodystrophy can involve the nail matrix or nail bed, which causes aesthetic and functional impairment.

8. Patients with psoriasis are at increased risk of a variety of medical conditions such as depression, anxiety, inflammatory bowel disease, malignancy, metabolic syndrome and cardiovascular disease.

9. All patients with generalised pustular psoriasis and erythrodermic psoriasis need urgent referral to the emergency department for admission.

10. An individualised treatment plan is required after incorporating the patient's preferences, the disease's impact on quality of life, and the potential benefits and adverse effects of therapies.

11. The severity of psoriasis can be determined by estimating the body surface area involved.

12. Patients with mild to moderate psoriasis can often be treated successfully with topical therapies.

13. For local, persistent lesions, potent topical steroids can be used in combination with a vitamin $\mathrm{D}$ analogue.

14. Phototherapy is a first-line treatment for patients with mild psoriasis.

15. For moderate to severe psoriasis, systemic therapies such as acitretin, methotrexate, cyclosporine and biologics may be considered.

16. Nonsteroidal anti-inflammatory drugs are the mainstay treatment of all patients with psoriatic arthritis.

17. Clinical characteristics of psoriatic arthritis include asymmetrical joint involvement, distal interphalangeal joint involvement, dactylitis and enthesopathy.

18. Patients with psoriatic arthritis require referral to an orthopaedic surgeon for further management.

19. Receiving regular advice on weight management, alcohol and smoking cessation can reduce cardiovascular risk factors among patients with psoriasis.

20. Moderate cases of psoriasis that are resistant to topical therapies, severe or stable erythrodermic psoriasis as well as psoriatic arthritis should be considered for early referral to specialists.

\begin{tabular}{|c|c|}
\hline True & False \\
\hline$\square$ & $\square$ \\
\hline$\square$ & $\square$ \\
\hline$\square$ & $\square$ \\
\hline$\square$ & $\square$ \\
\hline$\square$ & $\square$ \\
\hline$\square$ & $\square$ \\
\hline$\square$ & $\square$ \\
\hline$\square$ & $\square$ \\
\hline$\square$ & $\square$ \\
\hline$\square$ & $\square$ \\
\hline$\square$ & $\square$ \\
\hline$\square$ & $\square$ \\
\hline$\square$ & $\square$ \\
\hline$\square$ & $\square$ \\
\hline$\square$ & $\square$ \\
\hline$\square$ & $\square$ \\
\hline$\square$ & $\square$ \\
\hline$\square$ & $\square$ \\
\hline$\square$ & $\square$ \\
\hline$\square$ & $\square$ \\
\hline
\end{tabular}

\section{Doctor's particulars:}

Name in full:

MCR no.:

Specialty:

Email:

\section{SUBMISSION INSTRUCTIONS:}

Visit the SMJ website: http://www.smj.org.sg/current-issue and select the appropriate quiz. You will be redirected to the SMA login page.

For SMA member: (1) Log in with your username and password (if you do not know your password, please click on 'Forgot your password?'). (2) Select your answers for each quiz and click 'Submit'.

For non-SMA member: (1) Create an SMJ CME account, or log in with your SMJ CME username and password (for returning users). (2) Make payment of SGD 21.40 (inclusive of $7 \%$ GST) via PayPal to access this month's quizzes. (3) Select your answers for each quiz and click 'Submit'.

RESULTS:

(1) Answers will be published online in the SMJ May 2021 issue. (2) The MCR numbers of successful candidates will be posted online at the SMJ website by 10 May 2021. (3) Passing mark is $60 \%$. No mark will be deducted for incorrect answers. (4) The SMJ editorial office will submit the list of successful candidates to the Singapore Medical Council. (5) One CME point is awarded for successful candidates. (6) SMC credits CME points according to the month of publication of the CME article (i.e. points awarded for a quiz published in the March 2021 issue will be credited for the month of March 2021, even if the deadline is in May 2021).

Deadline for submission (March 2021 SMJ 3B CME programme): 12 noon, 3 May 2021. 